\title{
The Effect of Metabolism-Boosting Beverages on 24-hr Energy Expendi- ture
}

\author{
L.M. Davis*, ${ }^{*}$, C.D. Coleman ${ }^{1}$, W.S. Andersen ${ }^{1}$, L.J. Cheskin ${ }^{2}$ \\ ${ }^{1}$ Medifast Inc., Research \& Development, 11445 Cronhill Drive, Owings Mills, MD 21117, USA \\ ${ }^{2}$ Johns Hopkins Bloomberg School of Public Health, Department of International Health, Division of Human Nutrition, \\ Baltimore, MD 21205-2179, USA
}

\begin{abstract}
The effect of thermogenic meal replacement beverages (TMRB) containing $90 \mathrm{mg}$ EGCG and $100 \mathrm{mg}$ of caffeine on resting energy expenditure (REE) was tested. Thirty adults (19 women, 11 men) were stratified into 3 groups: lean ( $\mathrm{n}=10$, BMI $21.5 \pm 2.1$ ); overweight/obese (OW) $(\mathrm{n}=10$, BMI $29.8 \pm 2.7)$; or weight maintainers (WM) ( $=10$, BMI $28.8 \pm 4.0$ ). Following an overnight fast, baseline measurements, including REE via indirect calorimetry, were performed. REE was repeated at 30,60, 90, and 120 minutes after consuming a TMRB. Appetite was assessed via visual analogue scale at baseline, 30 minutes and 120 minutes after the TMRB. Mean 24-hour REE was increased $5.9 \pm 2.5 \%$ overall $(\mathrm{p}=0.000), 5.7 \pm 3.1 \%$ among lean subjects $(\mathrm{p}=0.0002), 5.3 \pm 1.4 \%$ among OW subjects $(\mathrm{p}=0.000)$, and $6.8 \pm 2.7 \%$ among $\mathrm{WM}(\mathrm{p}=0.0007)$. Appetite was significantly reduced 30 minutes after the TMRB ( $=0.0002)$. TMRB appear to be a promising weight control tool.
\end{abstract}

Keywords: Obesity, metabolism, weight loss, energy expenditure, thermogenesis.

\section{INTRODUCTION}

Obesity is a chronic, complex, multifactorial disorder [1] that has reached epidemic proportions in the United States. According to 2002 National Health and Nutrition Examination Survey [2] 65.7\% of adults are overweight and 30.6\% are characterized obese. Obesity is associated with an increased risk of heart disease, diabetes, cancer, asthma, sleep apnea, arthritis, reproductive problems, and psychological disturbances [3]. Despite the widespread prevalence of obesity and its and detrimental ramifications, effective treatment options remain scarce. Finding new weight control tools and strategies to help individuals gain short- and long-term control over their weight and health may help to curb this public health crisis.

One recent promising concept is the use of functional foods that burn extra calories. Calorie-burning, or thermogenic foods, such as green tea and cayenne pepper, when combined with caffeine, have been shown to increase resting energy expenditure by 3 to $8 \%[4,5]$.

Total energy expenditure at rest consists of three main components: approximately $60 \%$ is derived from basal metabolic rate; $30 \%$ is derived from physical activity level, and approximately $10 \%$ is derived from the thermic effect of food (TEF) [6]. TEF consists of two parts. One component of TEF is the result of energy required to digest, absorb and assimilate food nutrients, known as obligatory thermogenesis. The other component, facultative thermogenesis, is the result of food ingestion activating the sympathetic nervous system, with its stimulating effect on metabolism [7].

*Address correspondence to this author at the Medifast Inc., Research \& Development, 11445 Cronhill Drive, Owings Mills, MD 21117, USA; Tel: 410-504-8220; Fax: 410-504-8221; E-mail: ldavis@ choosemedifast.com
TEF has been shown to be blunted in overweight and obese individuals, compared to lean individuals [8]. Other risk factors for a blunted TEF include abnormal glucose tolerance [9], insulin resistance [10], visceral body fat distribution [11], and female gender [8]. TEF has been shown to be blunted in obese men [12]; however, compared to women, men have been shown to have a $25 \%$ greater TEF. In addition, during energy restriction while dieting, energy expenditure decreases, at least in the short-term [13]. Thus, the consumption of thermogenic foods may help to normalize energy metabolism among individuals at risk of having a blunted TEF, or who are actively attempting weight loss through low calorie dieting.

The combination of caffeine and epigallicocatechin gallate (EGCG), the active component in green tea, has been shown to stimulate an overall increase in energy metabolism after a period of weight loss and maintenance especially among low caffeine consumers [14]. Other potential weight control benefits of EGCG include: inhibition of the primary triglyceride-making enzyme, fatty acid synthetase [15], and a shift toward fatty acid oxidation [16].

By itself, caffeine appears to be a safe thermogenic agent for weight control that affects thermogenesis by inhibiting the phosphodiesterase-induced degradation of intracellular cyclic AMP [17]. Further, reduced food intake following caffeine has been demonstrated [18], suggesting that caffeine can affect both energy intake and energy expenditure. According to Rumpler et al. [19], the maximal increase in 24hour energy expenditure with caffeine, $3-7.2 \%$, occurs at doses of 200-300 mg per day. More than $400 \mathrm{mg}$ of caffeine begins to increase systolic and diastolic blood pressure and is associated with palpitations, anxiety, and dizziness compared to placebo [20]. Though acute caffeine consumption may alter some cardiovascular variables, chronic ingestion of caffeine has been shown to have little or no adverse health consequences [21]. 
Green tea has been widely consumed in China and Japan for centuries and is likely quite safe [21]. Further, green tea has potent antioxidant effects that are protective against oxidative stress, a condition that is increasingly recognized as a key mediator of many chronic diseases, including cancer and cardiovascular disease [22]. Green tea contains high quantities of several polyphenols, such as epicatechin, epicatechin gallate, epigallocatechin, and the most pharmacologically potent, EGCG. Catechins in green tea may stimulate thermogenesis and fat oxidation through inhibition of catecholO-methyl transferase (COMT), the enzyme that degrades norepinephrine [23].

Green tea and caffeine work synergistically to stimulate thermogenesis via COMT and phophodiesterase, respectively [24]. The synergistic relationship between EGCG and caffeine may be related, in part, to the overlapping timing of their peak concentrations and half-lives. Peak concentrations for caffeine occur at 30-60 minutes after consumption with doses ranging from 50 to $200 \mathrm{mg}$ [25], and at 1.5 to 2 hours for decaffeinated green tea solids containing EGCG. The half-lives of caffeine and EGCG, 5.7 hours [26] and 5 to 5.5 hours [27], respectively, are very similar as well.

Given the potential synergistic effect of EGCG and caffeine on energy metabolism, we sought to evaluate the effectiveness of thermogenic meal replacement beverages (TMRB) containing EGCG and caffeine on resting energy expenditure. The secondary hypothesis was to evaluate the effect of the TMRB on fat oxidation and appetite.

\section{MATERIALS AND METHODOLOGY}

\section{Subjects}

30 healthy adults (19 women, 11 men) between the ages of 18 and 65 years were recruited through flyers posted at local businesses and gyms. They were stratified into 3 groups: lean $(\mathrm{n}=10$, BMI <25); overweight/obese (OW) $(\mathrm{n}=10$, BMI $\geq 25)$; or weight maintainers (WM) $(\mathrm{n}=10)$. Baseline characteristics are contained in Table 1. Weight maintainers were subjects who had maintained a weight loss of $\geq 5 \%$ for at least a 3 -month period prior to the start of the study. Exclusion criteria included consumption of $>1$ cup of coffee (or equivalent) per day, current cigarette smoking, consuming $\geq 14$ alcoholic beverages per week (or any the day prior to study days), chronic uncontrolled health problems (not including obesity or diabetes); drug or alcohol dependence, mental illness (schizophrenia, bipolar disorder, current major depressive disorder), taking medications that would affect appetite or metabolism (e.g. steroids, Ritalin); active dieting; pregnancy or lactation; and allergy to wheat, gluten, soy or nuts.

\section{Methods}

Written, informed consent was obtained from all subjects under the auspices of the Western Institutional Review Board. After an overnight fast, subjects had the following baseline measurements performed: resting energy expenditure (REE), height, weight, waist circumference, blood pressure, and pulse. REE was measured by a SensorMedics Vmax29 Series Model V29 indirect calorimeter (SensorMedics Corporation, Yorba Linda, California) [28]. BMI was calculated using $\mathrm{kg} / \mathrm{m}^{2}$. Subjects received one of the six flavored TMRB (Momentum by Medifast, Inc, Owings Mills, MD). All six flavor versions contained 90-100 calories, 1114 grams of protein, 0-0.5 grams of fat, 3-4 grams of fiber, $90 \mathrm{mg}$ of EGCG and $100 \mathrm{mg}$ caffeine. REE was repeated at 30, 60, 90, and 120 minutes after consuming a single TMRB. In addition, to assess appetite effects, a visual analogue scale anchored by "not hungry at all" and "extremely hungry" was performed at baseline, and 30 and 120-minutes after consuming the TMRB.

Table 1. Baseline Characteristics Among Lean, OW, and WM Groups

\begin{tabular}{|c|c|c|c|}
\hline BMI $\left(\mathrm{kg} / \mathrm{m}^{2}\right)$ & $21.5 \pm 2.1$ & $29.8 \pm 2.7$ & $28.8 \pm 4.0$ \\
\hline \multicolumn{4}{|l|}{ Gender $(\%)$} \\
\hline Male & 10 & 50 & 50 \\
\hline \multicolumn{4}{|l|}{ Race $(\%)$} \\
\hline Caucasian & 70 & 50 & 90 \\
\hline African American & 0 & 40 & 10 \\
\hline Asian & 30 & 0 & 0 \\
\hline Hispanic & 0 & 10 & 0 \\
\hline \multicolumn{4}{|l|}{ Blood Pressure (mm Hg) } \\
\hline Pulse (beats per min) & $68.3 \pm 9.3$ & $67.6 \pm 13.0$ & $70.6 \pm 9.1$ \\
\hline Waist Circumference (in) & $29.6 \pm 2.9$ & $37.6 \pm 4.0$ & $36.8 \pm 4.0$ \\
\hline$\%$ Physically active (exercise $\geq 3$ hours per wk) & 75 & 78 & 75 \\
\hline
\end{tabular}

Results are reported as either a (mean $\pm \mathrm{sd}$ ) or as a percentage. 


\section{Statistical Analysis}

All data were collected using a standardized format, entered into Microsoft Excel, and then exported to the STATA statistical software package (College Station, TX) [29] for analysis. Means \pm standard deviation scores or percentages were calculated for all baseline demographic and clinical variables. Data distributions were examined and outliers were evaluated. Main outcome variables (REE and fat oxidation) were evaluated using paired sample t-tests. Repeated measures analysis of variance (RANOVA) was used to evaluate change in appetite over time. Linear regression analysis was performed to examine associations between outcome variables and subject characteristics. Data were considered statistically significant if $\mathrm{p}<0.05$.

\section{RESULTS}

Overall, mean REE increased by $24.2 \pm 10.1 \%$ for the 2 hour period following the consumption of a TMRB compared to baseline $(\mathrm{p}=0.000)$. Compared to baseline, mean REE for the 2-hour period following a TMRB was increased $22.8 \pm 12.4 \%$ among lean $(\mathrm{p}=0.00015), 21.6 \pm 6.2 \%$ among OW ( $\mathrm{p}=0.000)$, and $27.2 \pm 10.9 \%$ among WM individuals $(\mathrm{p}=0.00016)$ (Fig. 1). When extrapolated over the 24-hour period, based on our recommendation of consuming 3 TMRBs per day, the mean increase in 24-hour REE among all subjects was $5.9 \pm 2.5 \%$. Mean 24-hour REE increased by
$5.7 \pm 3.1 \%$ among lean, $5.3 \pm 1.4 \%$ among $\mathrm{OW}$, and $6.8 \pm$ $2.7 \%$ among WM individuals. There was an overall trend toward increased fat oxidation with RQ decreasing from 0.99 \pm 0.19 to $0.92 \pm 0.13, p=0.122$. While significant reductions were not observed among the OW or WM groups, a significant reduction in RQ from $1.0 \pm 0.165$ to $0.915 \pm 0.04$ was found among lean individuals $(\mathrm{p}=0.01)$. Using RANOVA, a significant change in appetite was observed over time in response to the TMRB [F=9.7, $\mathrm{p}=0.0002]$. Pairwise comparisons revealed a significant overall reduction in appetite at the 30 -minute time point after consuming the TMRB $(5.7 \pm 2.7$ vs. $3.8 \pm 2.3, \mathrm{p}=0.0002)$ that did not remain significant at the 120 minute time point $(5.6 \pm 2.8$ vs. $5.8 \pm 2.9, \mathrm{p}=0.71)$. A trend for group differences between lean, overweight, and weight maintainers $[\mathrm{F}=2.43, \mathrm{p}=0.095]$ was observed. Pairwise comparisons revealed significant reductions in appetite among the lean $(5.8 \pm 2.8 v s .4 .35 \pm 2.6, \mathrm{p}=0.015)$, WM (6.2 \pm 2.15 vs. $4.2 \pm 1.7, \mathrm{p}=0.025)$, and marginally among the OW groups $(5.1 \pm 3.3 v$ s. $2.8 \pm 2.5, \mathrm{p}=0.056)$ at 30 minutes. None of the groups sustained significant reductions in appetite at 120 minutes. According to linear regression analysis, no significant associations were found between the main outcome variables (REE, fat oxidation, or appetite) and weight category (lean, overweight, weight maintainer) or gender. No adverse events were reported at any time during this study.

\section{Effect of TMRB on \\ Resting Energy Expenditure (REE) \\ (avg. increase in REE for 2 hour period after consuming TMRB)}

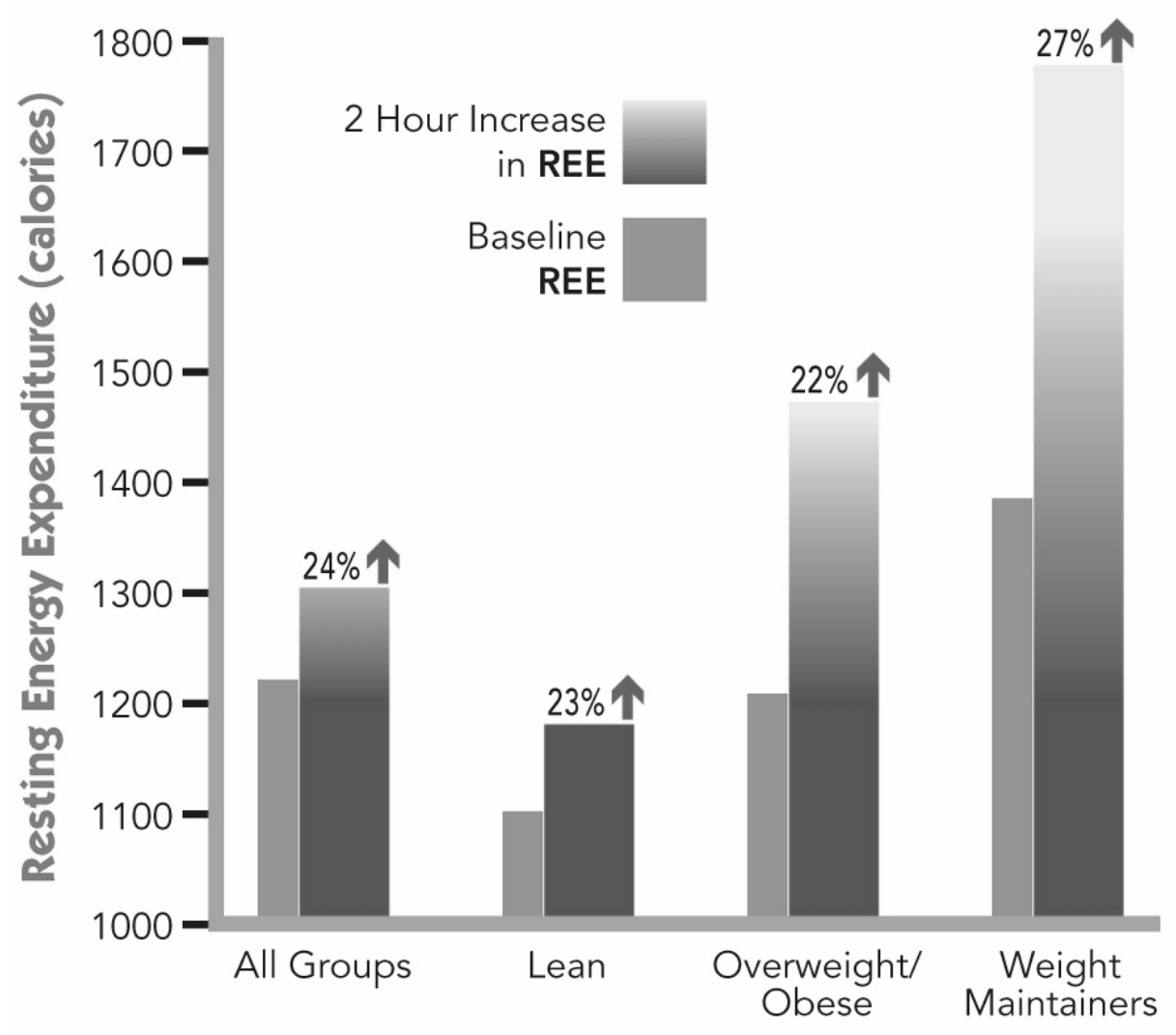

Fig. (1). 


\section{DISCUSSION}

The most salient result of this study was the robust increase in REE of nearly $25 \%$ among all subjects for the 2hour period following consumption of the TMRB. Extrapolated over the 24-hour period, this is equivalent to an approximately $6 \%$ increase in REE. Our results are consistent with that of others examining the effects of green tea components on energy metabolism. Several human clinical trials support a synergistic effect of caffeine and green tea on increasing total energy expenditure via thermogenesis. Dulloo et al. [30] demonstrated that green tea extract $(90 \mathrm{mg})+$ caffeine $(100 \mathrm{mg})$ three times per day with meals resulted in a significant increase in 24-hour energy expenditure (78 calories) and a significant decrease in respiratory quotient (indicating greater fat oxidation), compared with placebo. Berube-Parent et al. [4] evaluated the effect of a capsule of $200 \mathrm{mg}$ caffeine + either 90, 200, 300, or $400 \mathrm{mg}$ EGCG three times daily before meals on 14 healthy, non-smoking, sedentary men in a randomized placebo-controlled crossover trial. 24-hour energy expenditure was significantly increased at all doses of EGCG. They also evaluated EGCGcaffeine mixtures plus guarana, and found that all significantly increased 24-hour energy expenditure by $750 \mathrm{~kJ}$ ( 160 kcal) (8\%). Diepvens et al. [31] performed a randomized, double-blind, placebo controlled trial to examine the effect of green tea extract, along with a low energy diet, on REE in 46 overweight women. REE, as a function of fat-free mass, was significantly reduced over the 32-day study period in the placebo group, but not the group receiving green tea (1125 mg tea catechins $+225 \mathrm{mg}$ caffeine/day). Komatsu et al. [32] conducted a randomized, placebo-controlled, crossover trial in 11 healthy females and demonstrated that a single green tea drink containing $156 \mathrm{mg}$ EGCG + $161 \mathrm{mg}$ caffeine and $17 \mathrm{mg}$ polymerized polyphenols increased energy expenditure by $13 \mathrm{kcal} / 2$ hours (5\%), whereas oolong tea, containing $81 \mathrm{mg}$ EGCG + $77 \mathrm{mg}$ caffeine, and $68 \mathrm{mg}$ polymerized polyphenols, increased energy expenditure $26 \mathrm{kcal}$ / 2hours (10\%). Similarly, Rumpler et al. [19] showed that oolong tea (244 mg EGCG + $270 \mathrm{mg}$ caffeine) increased energy expenditure in healthy men $2.9 \%$, for an additional $281 \mathrm{kcal} / \mathrm{d}$ over a 3 -day period, versus $3.4 \%(331 \mathrm{kcal} / \mathrm{d})$ for a $(270 \mathrm{mg} / \mathrm{d})$ caffeinated water drink.

We found the greatest change in REE after consuming the TMRB among the weight maintainers. Other studies evaluating similar doses of EGCG and caffeine among overweight weight maintainers also found significant increases in energy expenditure. Westerterp-Plantenga, et al. [14] conducted a randomized, placebo-controlled, double blind study in 76 overweight/obese subjects to evaluate a very low energy diet for 4 weeks, followed by 3 months of weight maintenance where a green tea-caffeine mixture (6 capsules/day totaling $270 \mathrm{mg}$ EGCG + $150 \mathrm{mg}$ caffeine) was provided, versus placebo. They found that individuals who consumed low amounts of caffeine/day ( $<300 \mathrm{mg} /$ day) typically significantly increased their energy expenditure and had a positive effect on weight loss and weight maintenance. Subjects had followed a low energy diet for 4 weeks followed by a 3 months of weight maintenance.

Other potential weight control benefits of EGCG include inhibition of fatty acid synthetase [15], the primary triglyceride-making enzyme, and a shift toward fatty acid oxidation
[16]. Our study demonstrated an overall decrease in RQ, which is an indicator of increased fatty acid oxidation, as well as a significant appetite suppressing affect over the short-term in response to the TMRB.

There were a few limitations of the study worth noting. The first limitation was the extrapolation of single meal thermogenic results to 3-meals to estimate the effects over a 24hour time period. The second limitation was the lack of sufficient power needed to detect differences at the group level when clinically relevant trends emerged between lean, overweight, and weight maintaining individuals. This study was primarily designed to be a proof of concept study so was powered to detect outcome measure differences for the group as a whole. The third study limitation was the use of the one sample pre-test/post-test design. Despite the inherent limitations of this design, we felt that potential threats to validity were well-controlled as each person served as their own control, the study took place in a controlled environment, and it was conducted over a short period of time. These controls make it unlikely that factors other than the effect of the TMRB itself affected the study outcomes. Also, despite our relatively small sample size, selection bias was minimized by our recruitment of men and women with a range of different body weights, ethnicities and demographics.

\section{CONCLUSION}

Weight regain after weight loss is a nearly universal phenomenon. Thermogenic foods may help to offset this weight re-gain. TMRB may be most appropriate for patients during the maintenance phase of a diet plan as a means to prevent weight re-gain. If they saved $\sim 100$ calories per day by consuming 3 thermogenic products, patients would potentially prevent a weight regain of 10 pounds per year.

\section{ABBREVIATIONS}

$$
\begin{aligned}
& \mathrm{OW}=\text { Overweight/obese } \\
& \mathrm{TMRB}=\text { Thermogenic meal replacement beverages } \\
& \mathrm{WM}=\text { Weight maintainer }
\end{aligned}
$$

\section{ACKNOWLEDGEMENTS}

This study was funded as a program evaluation by a Medifast Inc.

\section{REFERENCES}

[1] Thearle M, Aronne LJ. Obesity and pharmacologic therapy. Endocrinol Metab Clin North Am 2003; 32: 1005-24.

[2] Hedley AA, Ogden CL, Johnson CL, Carroll M, Curtin LR, Flegal KM. Prevalence of overweight and obesity among US children, adolescents, adults, 1999-2002. JAMA 2004; 291: 2847-50.

[3] Gale SM, Castracane VD, Mantzoros CS. Energy homeostasis, obesity, and eating disorders: recent advances in endocrinology. $\mathrm{J}$ Nutr 2004; 134: 295-8.

[4] Berube-Parent S, Pelletier C, Dore J, Tremblay A. Effects of encapsulated green tea and Guarana extracts containing a mixture of epigallocatechin-3-gallate and caffeine on $24 \mathrm{~h}$ energy expenditure and fat oxidation in men. Br J Nutr 2005; 94: 432-6.

[5] Yoshioka M, Doucet E, Drapeau V, Dionne I, Tremblay A. Combined effects of red pepper and caffeine consumption on $24 \mathrm{~h}$ energy balance in subjects given free access to foods. Br J Nutr 2001; 85: 203-11

[6] Segal KR, Presta E, Gutin B. Thermic effect of food during graded exercise in normal weight and obese men. Am J Clin Nutr 1984; 40: 995-1000.

[7] Acheson KJ, Schutz Y, Bessard T, Anantharaman K, Flatt J-P, Jequier E. Glycogen storage capacity and de novo lipogenesis dur- 
ing massive carbohydrate overfeeding in man. Am J Clin Nutr 1988; 48: 240-7.

[8] Gougeon R, Harrigan K, Tremblay J-F, Hedrei P, Lamarche M, Morais JA. Increase in the thermic effect of food in women by adrenergic amines extracted from citrus aurantium. Obes Res 2005; 13: 1187-94

[9] Golay A, Schutz Y, Meyer HU, et al. glucose-induced thermogenesis in nondiabetic and diabetic obese subjects. Diabetes 1982; 31: 1023-8.

[10] Segal KR, Albu J, Chun A, Edano A, Legaspi B, Pi-Sunyer FX. Independent effects of obesity and insulin resistance on postprandial thermogenesis in men. J Clin Invest 1992; 89: 824-33.

[11] den Besten C, Vansant G, Westrate JA, Deurenberg P. Resting metabolic rate and diet-induced thermogenesis in abdominal and gluteal-femoral obese women before and after weight reduction. Am J Clin Nutr 1988; 47: 840-7.

[12] Segal KR, Gutin B, Nyman AM, Pi-Sunyer FX. Thermic effect of food at rest, during exercise, and after exercise in lean and obese men of similar body weight. J Clin Invest 1985; 76: 1107-1112.

[13] Connollly J, Romano T, Patruno M. Effects of dieting and exercise on resting metabolic rate and implications for weight management. Fam Pract 1999; 16: 196-201

[14] Westerterp-Plantenga MS, Lejeune MPGM, Kovacs EMR. Body weight loss and weight maintenance in relation to habitual caffeine intake and green tea supplementation. Obes Res 2005; 13: 11951204.

[15] Riorini, RN, Donovan JL, Rodwell D, et al. Short-term administration of (-)-epigallocatechin gallate reduces hepatic steatosis and protects against warm hepatic ischemia/reperfusion injury in steatotic mice. Liver Transpl 2005; 11: 298-308.

[16] Klaus S, Pultz S, Thone-Reineke C, Wolfram S. Epigallocatechin gallate attenuates diet-induced obesity in mice by decreasing energy absorption and increasing fat oxidation. Int J Obes 2005; 29: 615-623.

[17] Dulloo AG. Ephedrine, xanthines and prostaglandin-inhibitors: actions and interactions in the stimulation of thermogenesis. Int $\mathbf{J}$ Obes Relat Metab Disord 1993; 17(Suppl 1): S35-40.

[18] Racotta IS, Leblanc J, Richard D. The effect of caffeine on food intake in rats: involvement of coricotropin-releasing factor and the sympatho-adrenal system. Pharmacol Biochem Behav 1994; 48: 887-92.

[19] Rumpler W, Seale J, Clevidence B, et al. Oolong tea increases metabolic rate and fat oxidation in men. J Nutr 2001; 131: 2848-52.
[20] Astrup A, Toubro S, Cannon S, Hein P, Breum L, Madsen J. Caffeine: a double-blind, placebo-controlled study of its thermogenic, metabolic, and cardiovascular effects in healthy young volunteers. Am J Clin Nutr 1990; 51: 759-67.

[21] Diepvens K, Westerterp K, Westerterp-Plantenga MS. Obesity and thermogenesis related to the consumption of caffeine, ephedrine, capsaicin and green tea. Am J Physiol Regul Integr Comp Physio 2007; 292: R77-85.

[22] Keaney Jr JF, Larson MG, Vasan RS, et al. Obesity and Systemic Oxidative Stress. Arterioscler Thromb Vasc Biol 2003; 23: 434-9.

[23] Borchardt TR, Huber JA. Catechol O-methyltransferase. Structureactivity relationships for inhibition by flavonoids. J Med Chem 1975; 18: 120-122.

[24] Dulloo AG, Seydoux J, Girardier L, Chantre P, Vandermander J. Green tea and thermogenesis: interactions between catechinpolyphenols, caffeine, and sympathetic activity. Int J Obes 2000; 24: $252-28$

[25] Syed SA, Kamimori GH, Kelly W, Eddington ND. Mutliple dose pharmacokinetics of caffeine administered in chewing gum to normal healthly volunteers. Biopharm Drug Dispos 2005; 26: 403-9.

[26] Statland BE, Demas TJ. Serum caffeine half-lives. Healthy subjects vs. patients having alcoholic hepatic disease. Am J Clin Pathol 1980; 73: 390-3.

[27] Yang CS, Chen L, Lee MJ, Balentine D, Kuo MC, Schantz SP. Blood and urine levels of tea catechins after ingestion of different amounts of green tea by human volunteers. Cancer Epidemiol Biomarkers Prev 1998; 7: 351-4.

[28] SensorMedics Vmax29 Series, Model V29: SensorMedics Corporation: Yorba Linda, CA

[29] Stata Statistical Software: release 7.0 College Station TX. Stata Corporation: College Station, TX 1994-2001.

[30] Dulloo AG, Duret C, Rohrer D, et al. Efficacy of a green tea extract rich in catechin polyphenols and caffeine in increasing 24-h energy expenditure and fat oxidation in humans. Am J Clin Nutr 1999; 49: 44-50.

[31] Diepvens K, Kovacs EM, Nijs IM, Vogels N, Westerterp-Plantenga MS. Effect of green tea on resting energy expenditure and substrate oxidation during weight loss in overweight females. $\mathrm{Br} \mathrm{J}$ Nutr 2005; 94: 1026-34.

[32] Komatsu T, Nakamori M, Komatsu K, et al. Oolong tea increases metabolism in Japanese females. J Med Invest 2003; 50: 170-5. 